Raman Spectroscopic Analysis of Biochemical Changes in Individual Triglyceride-Rich Lipoproteins in the Preand Postprandial State

J. Chan, D. Motton, J. Rutledge, N. Keim, T. Huser

September 16, 2004

Analytical Chemistry 
This document was prepared as an account of work sponsored by an agency of the United States Government. Neither the United States Government nor the University of California nor any of their employees, makes any warranty, express or implied, or assumes any legal liability or responsibility for the accuracy, completeness, or usefulness of any information, apparatus, product, or process disclosed, or represents that its use would not infringe privately owned rights. Reference herein to any specific commercial product, process, or service by trade name, trademark, manufacturer, or otherwise, does not necessarily constitute or imply its endorsement, recommendation, or favoring by the United States Government or the University of California. The views and opinions of authors expressed herein do not necessarily state or reflect those of the United States Government or the University of California, and shall not be used for advertising or product endorsement purposes. 
UCRL-JRNL-206592

\title{
Raman Spectroscopic Analysis of Biochemical Changes in Individual Triglyceride-Rich Lipoproteins in the Pre- and Postprandial State
}

\author{
J.W. Chan ${ }^{1,3^{*}}$, D. Motton ${ }^{2}$, J.C. Rutledge ${ }^{2,3}$, N. L. Keim ${ }^{4}$, and T. Huser ${ }^{1,3}$ \\ 1.Lawrence Livermore National Laboratory, 7000 East Ave, Livermore, CA 94550 \\ 2.School of Medicine - Department of Internal Medicine, University of California, \\ Davis, CA, 95616 \\ 3.NSF Center for Biophotonics Science and Technology, University of California, \\ Davis, Sacramento, CA, 95817 \\ 4.Western Human Nutrition Research Center, U.S. Department of Agriculture, \\ Davis, CA, 95616
}

*Corresponding author: chan19@\|lnl.gov, fax: (925) 424-2778

Keywords: hyperlipemia, lipids, lipoproteins, Raman spectroscopy, biophotonics, laser tweezers

\begin{abstract}
Individual triglyceride-rich lipoprotein (TGRL) particles derived from human volunteers are non-destructively analyzed by laser tweezers Raman microspectroscopy and information on their composition and distribution is obtained. The Raman signature of single optically trapped very low-density lipoproteins (VLDL), a subclass of TGRL, which play an important role in cardiovascular disease, exhibits distinct peaks associated with molecular vibrations of fatty acids, proteins, lipids, and structural rearrangements of lipids. Our analysis of pre- and postprandial VLDL exhibits the signature of biochemical changes in individual lipoprotein particles following the consumption of meals. Interaction of VLDL with endothelium leads to the breakdown of complex triacylglycerols and the formation of a highly ordered core of free saturated fatty acids in the particle. A particle distribution analysis reveals trends in the degree to which this process has occurred in particles at different times during the postprandial period.
\end{abstract}


Differences in particle distributions based on the different ratios of polyunsaturated to saturated fats in the consumed meals are also easily discerned. Individual lipoprotein particles hydrolyzed in-vitro through addition of lipoprotein lipase (LpL) exhibit strikingly similar changes in their Raman spectra. These results demonstrate the feasibility of monitoring the dynamics of lipid metabolism of individual TGRL particles as they interact with LpL in the endothelial cell wall using Raman spectroscopy.

\section{Introduction}

Lipoproteins are ten nanometer to one micron diameter lipid particles found in blood that are responsible for the transport of fatty acids and cholesterol as fuel for cells throughout the body. Lipoproteins consist of a monolayer shell of phospholipids, cholesterol, and apoproteins enveloping a hydrophobic core of triglycerides and cholesterol esters ${ }^{1}$. Triglyceride-rich lipoproteins (TGRL) in blood are generated from an exogenous pathway (chylomicrons) and an endogenous pathway (very low-density lipoproteins; VLDL). Elevation of blood lipid levels after the consumption of a meal (postprandial hyperlipemia) is the result of contributions from both of these pathways. Once in the blood, postprandial lipoproteins are hydrolyzed by an enzyme anchored to endothelial cells, lipoprotein lipase ( $L \mathrm{pL})$, to form smaller remnant particles and other lipolysis products, such as free fatty acids. The composition and relative distribution of TGRL are implicated in the onset of atherosclerotic cardiovascular disease, the leading cause of morbidity and mortality in the U.S. and most of the 
Western World. These lipoprotein particles are known to penetrate the arterial wall and become trapped, initiating the early stages of atherosclerotic lesions. Therefore, acquiring information on the structure of lipoprotein particles in the pre- and postprandial states, as well as changes due to interactions with enzymes expressed on endothelial cells, will assist in elucidating the pathogenesis of atherosclerosis.

Previous studies using postprandial TGRL have primarily used standard ultracentrifugation and biochemical analysis techniques of bulk samples. These types of studies investigate entire populations of lipoproteins at a time and are therefore not specific to chemical differences between individual particles. Here, we demonstrate that a novel technique combining laser tweezers with confocal micro-Raman spectroscopy allows for the non-invasive, non-destructive analysis of individual TGRL in their native environment. Raman spectroscopy is a laserbased vibrational spectroscopy technique for the analysis of molecular bonds due to inelastic scattering of photons. Raman-scattered photons are scattered predominantly with a shift towards longer wavelengths (Stokes-shifted), whereby they deposit parts of their energy in the sample. The difference between the incident and scattered frequencies corresponds to the energy of the molecular vibration. Therefore, a Raman spectrum is a "molecular fingerprint" of the sample, providing a wealth of information about the chemical bonds associated with DNA, proteins, lipids, and carbohydrates commonly found in biological samples $^{2}$. Changes in their composition thus reveal themselves as differences in the Raman spectrum. It has recently been shown that if Raman spectroscopy is 
combined with confocal microscopy, even individual cells or subcellular components can be analyzed ${ }^{3}$. Femtoliter sample volumes can be analyzed with a diffraction-limited spatial resolution of better than $1 \mu \mathrm{m}$.

Individual biological samples in the nanometer size regime that are unconstrained in their natural aqueous environment are usually difficult to probe by confocal Raman micro-spectroscopy. Typically, these particles need to be dried on the surface of a substrate, which may change their biological activity. Also, in this case, locating the nanometer-sized particles by scanning the substrate for Raman and/or fluorescence signals becomes extremely difficult, if not impossible, due to the background of the substrate and the minute particle size. By combining laser trapping with confocal Raman spectroscopy, such particles can be probed while naturally suspended in their native environment without the need for particle adhesion to a surface. Optical trapping in its simplest form involves the use of a single, tightly focused laser beam to immobilize a particle within the laser focus by optical forces ${ }^{4}$. Trapping is achieved as photons impinging on the particle transfer momentum from the light beam, imparting reaction forces on the object. The balance of both scattering and gradient forces on the object near the laser focus results in a stable three-dimensional optical trap. This technique, first pioneered by Ashkin and $\mathrm{Chu}^{5}$, has been used extensively in the biophysical sciences for the manipulation of biological samples (bacteria, viruses, cells, organelles) ${ }^{6-9}$ and the determination of biomolecular interaction forces ${ }^{10,}{ }^{11}$. Recent work has applied laser tweezers Raman spectroscopy to the vibrational analysis of biological particles suspended in 
solution $^{12,13}$ Recently, Xie and coworkers ${ }^{14-16}$, for example, demonstrated the application of optical trapping combined with Raman spectroscopy and shifted excitation Raman spectroscopy to study the dynamics of thermal denaturation of E. coli bacteria and yeast cells. Other recent work has also demonstrated the use of this method to analyze the composition and properties of individual liposomes ${ }^{17-19}$. This approach also allows for the determination of subpopulations in a mixed, heterogeneous sample ${ }^{12}$, an important parameter that cannot be obtained by conventional bulk spectroscopy.

The main goals of the present work are to determine to what level of detail information about the biochemical composition and biochemical changes can be extracted from single lipoproteins undergoing lipid metabolism. Such information can provide new insight into the chemical processes leading to atherosclerosis. In this study, a subgroup of TGRL particles, VLDL, are targeted for chemical analysis. Pre- and postprandial particles from human subjects consuming different diets are chemically characterized at different points in time during the postprandial state. We also determine biochemical changes in VLDL undergoing in-situ hydrolysis during exposure to lipoprotein lipase ( $L p L)$ and compare the results. In-situ hydrolysis simulates the repeated lipolysis of lipoprotein particles by lipoprotein lipase anchored to the endothelial cell layer of blood vessels. To date, no studies have analyzed the composition and biochemical changes of individual plasma-derived lipoprotein particles undergoing lipoprotein metabolism and investigated the statistical variations in the composition from particle to particle. Few studies have been aimed at the $\operatorname{Raman}^{20}$ or infrared (IR) 
spectroscopy ${ }^{21,22}$ of lipoprotein structure and they were all performed on bulk solutions of lipoproteins. Laser light scattering spectroscopy ${ }^{23}$ has yielded bulkaveraged information about the size and density of lipoproteins before and after the onset of ischemia. Here, we show that the bio-molecular Raman spectroscopic fingerprint of individual VLDL is unique, highly reproducible and can be used to monitor biochemical changes of the particles due to lipoprotein metabolism.

\section{Experimental Section}

Preparation of TGRL samples

Blood was obtained from human female volunteers participating in a clinical nutrition research study examining postprandial lipemia in response to meals with low and high glycemic loads. The study was approved by the Human Subjects Research Committee of the University of California, Davis, CA. All volunteers were overweight with BMI 27-29 and between 24-35 years of age. For 3 days prior to blood collection, volunteers consumed controlled diets with sufficient calories to match their individual energy requirements (2000-2200 kcal/d) and moderate fat content (30\%). Fasting blood was obtained after a $12-\mathrm{h}$ overnight fast. For the postprandial studies, volunteers consumed controlled mixed meals containing $30 \%$ total fat, $15 \%$ protein, and $55 \%$ carbohydrate. The mixed meal with the low glycemic load contained carbohydrates from whole grain products and a polyunsaturated:saturated $(P: S)$ fat ratio of 0.2 , whereas the high glycemic load meal contained carbohydrates from refined grain products and P:S ratio of 
0.10. Postprandial blood was drawn at 3.5 and $8 \mathrm{~h}$ following meal ingestion. Blood was collected in $10 \mathrm{ml}$ vacutainer tubes filled with streptokinase (150 units $/ \mathrm{ml}$ blood). Whole blood was then spun at $4^{\circ} \mathrm{C}$ for 10 minutes at $1750 \mathrm{~g}$, to remove cellular particulate. After centrifugation, plasma was transferred to ultracentrifuge tubes (Beckman-Coulter) and centrifuged at $285,000 \mathrm{~g}$ at $4^{\circ} \mathrm{C}$ for 4 hours to isolate TGRL. This procedure removes all higher density lipoprotein particles (LDL, HDL, and IDL) with a diameter of less then $40 \mathrm{~nm}$. After ultracentrifugation, TGRL was removed and the concentration was measured using an Infinity Triglyceride Concentration kit (Sigma Diagnostics). The final samples containing only chylomicrons, VLDLs and their remnants were stored on dry ice and then transported to LLNL. The plasma was diluted 1:100 in PBS buffer to separate and isolate individual TGRL in buffer solution. Analysis of the TGRL by LTRS was typically conducted no later than 48 hours after extraction.

Acquisition of Raman spectra of single optically trapped TGRL

The LTRS system consists of a $632.8 \mathrm{~nm}$ CW laser beam spectrally filtered with a $633 \mathrm{~nm}$ bandpass filter and delivered into the back port of a Zeiss Axiovert 200 inverted microscope. A longpass dichroic reflector directs the beam through a 100X, 1.3 NA oil immersion objective (Zeiss), resulting in a diffraction limited spot of roughly $0.5 \mu \mathrm{m}$ diameter with $10 \mathrm{~mW}$ laser power. The tight focus creates an optical trap that immbolizes single TGRL particles drifting in the plasma solution (Figure 1). White light illumination in transmission is used to obtain images captured on a CCD video camera. Epi-detection of the Raman signals of the 
trapped particle is achieved using the same objective and a $100 \mu \mathrm{m}$ pinhole in a confocal arrangement. The signals are filtered using a holographic $633 \mathrm{~nm}$ notch filter for suppression of residual laser light, directed into a spectrometer equipped with a 1200 lines $/ \mathrm{mm}$ grating, blazed at $500 \mathrm{~nm}$, and focused onto a liquid nitrogen cooled CCD camera $(1340 \times 100$ pixels). Optical trapping of single TGRL particles is accomplished by using the translation stage to move the particles to a close proximity of the laser focus, at which point the particle will be drawn into the focus and then trapped. A typical acquisition time of 60 seconds is sufficient to acquire a Raman spectrum of typically better than 10:1 signal-tonoise ratio with clearly defined Raman bands. Spectra are calibrated to a toluene standard and background correction is performed on each spectrum by subtraction of a $3^{\text {rd }}$ order polynomial baseline fit. Each spectrum is normalized to the intensity of the $1440 \mathrm{~cm}^{-1}$ peak because fluctuations due to changes in protein concentration or conformation are relatively weak. The differentiation of the differently sized TGRL (large vs. small) was based on their visibility in the optical microscope by comparison to polystyrene beads of well-known size.

\section{Acquisition of Raman spectra from unsaturated and saturated oils}

Cis-9-octadecenoic acid (oleic acid), hexadecanoic acid (palmitic acid) and octadecanoic acid (stearic acid) oil standards with purity greater than $95 \%$ were purchased from Sigma. The saturated fatty acid samples (palmitic acid and stearic acid are solid at room temperature. To simulate the effects of carbon chain disorderning, samples of palmitic and stearic acid were also heated above 
their melting point in a water bath. The samples were examined at room temperature and $\sim 70$ deg. $C$, respectively, by placing either the powder (palmitic and stearic acids at room temperature) or liquid (oleic acid; palmitic and stearic acid heated above the melting point) on a calcium fluoride substrate. A $20 \mathrm{X}$ long working distance objective is used to focus the $633 \mathrm{~nm}$ laser beam onto the samples. Spectra are acquired with a 30 second integration time.

Hydrolyzation of TGRL particles with lipoprotein lipase (LpL)

Lipoprotein lipase from bovine milk, (diacylglycerol acylhydrolase), was purchased from Sigma. $15 \mu \mathrm{L}$ of the enzyme is added to approximately $1 \mathrm{~mL}$ of TGRL particles in plasma solution in an Eppendorf tube, with a density of 400 $\mathrm{mg} / \mathrm{dL}$. This results in a concentration of $1.4 \times 10^{-2} \mathrm{mg} / \mathrm{mL}$ (74 total units) of enzymatic proteins. Spectra of many particles are taken both before and 60 minutes after addition of the LpL. Figure 5 shows a sample spectrum of individual particles before and after exposure to $L p L$ and the background from $L p L$ in buffer.

\section{Results and Discussion}

In this study, we analyze the biochemical changes that occur in VLDL during normal human lipid metabolism. Even though the subject samples after ultracentrifugation also contain chylomicrons, those can be easily distinguished from VLDLs due to their significant difference in size. An initial calibration of our optical system using polystyrene beads of known sizes in solution revealed that 
only beads larger than $\sim 150 \mathrm{~nm}$ can be visually observed using white light illumination in transmission and a 100X 1.3 NA oil immersion objective. We use this value as a benchmark to discriminate between the "small" VLDL and remnant particles and "large" chylomicron particles in our samples that range in size typically from 40 to $1000 \mathrm{~nm}$. We find that for particles of roughly $150 \mathrm{~nm}$ and larger, a single trapped TGRL at the focus can be observed by white light imaging with a CCD camera (not shown). For smaller particles (<100 nm; VLDL and remnant particles), optical trapping is still feasible with the laser power used in this study. In this case, the TGRL are detected by the Rayleigh-scattering of the laser light backscattered off the trapped particle (see the inset of figure 2), which can be observed after removal of the filters in front of the CCD camera. It should be noted that for smaller particles it is possible that a cluster of particles is trapped at the laser focus rather than a single particle although this is minimized by appropriate dilution of the particle solution. Also, clustering in the case of polystyrene test particles led to fluctuations in the backscattered light intensity, which we did not observe in the case of TGRLs. We feel therefore confident that we trapped individual TGRL for the duration of the Raman signal acquisition.

A typical background-corrected and normalized Raman spectrum of a single trapped TGRL particle is shown in Figure 2. This spectrum was obtained from a VLDL particle in plasma from a volunteer who consumed a controlled diet with moderate fat content (30\%) for 3 days and then fasted for 12 hours prior to sample extraction. We call this a $0 \mathrm{hr}$ sample, because it was obtained before the volunteers consumed a meal. The spectrum was obtained with a 60 second 
signal accumulation time using $10 \mathrm{~mW}$ of $633 \mathrm{~nm}$ laser power and shows a rich vibrational structure. The major peaks and their assignments are listed in Table I.

The peaks observed in this 0 hour spectrum of a VLDL particle can be readily assigned to known major lipid bands by comparing them to previously published results. Frank et. al ${ }^{24}$ have observed similar Raman bands in human breast tissue and studies on oils and fats have also observed similar spectra ${ }^{25,26}$. The peak at $727 \mathrm{~cm}^{-1}$ is assigned to an in-plane bending mode of the $\mathrm{C}-\mathrm{H}$ bond in a double bond. The $972 \mathrm{~cm}^{-1}$ peak is an out-of-plane deformation mode of the $\mathrm{C}-\mathrm{H}$ bond. The peaks at 1066,1076 , and $1129 \mathrm{~cm}^{-1}$ correspond to $\mathrm{C}-\mathrm{C}$ stretching vibrations and the $1266 \mathrm{~cm}^{-1}$ peak is due to an in-plane $\mathrm{C}-\mathrm{H}$ bending mode in a double bond. The peak at $1302 \mathrm{~cm}^{-1}$ can be attributed to a $\mathrm{CH}_{2}$ twisting mode, the $1439 \mathrm{~cm}^{-1}$ peak to a $\mathrm{CH}_{2}$ scissor mode, the $1654 \mathrm{~cm}^{-1}$ peak to a $\mathrm{C}=\mathrm{C}$ stretch mode (indicative of unsaturated bonds), and the $1742 \mathrm{~cm}^{-1}$ to a $\mathrm{C}=\mathrm{O}$ bond in an ester. The broad shoulder on both sides of the $1654 \mathrm{~cm}^{-1}$ band is a background contribution from the $\mathrm{O}-\mathrm{H}$ stretching vibration of water in the plasma solution. These peaks provide information about the chemical components and conformations of the VLDL. It is known ${ }^{25}$ that spectra of triacylglycerol exhibit the presence of the $1742 \mathrm{~cm}^{-1}$ peak that is lacking in spectra of free fatty acids, confirming that triacylglycerol is likely present in the core of the lipoprotein particle. The location of the $1650 \mathrm{~cm}^{-1}$ band indicates a cis conformation in the $C=C$ double bond, which would otherwise be located at $1668 \mathrm{~cm}^{-1}$ for the trans conformation, and is therefore another marker for unsaturated bonds. The presence of the $1266 \mathrm{~cm}^{-1}$ band also indicates a cis geometry. Raman bands due 
to apoprotein modes, however, are difficult to identify because of the overwhelming contributions from lipids. For example, the amide I band at 1655 $\mathrm{cm}^{-1}$ is not easily distinguished from the $1655 \mathrm{~cm}^{-1}$ lipid band and the broad water background band. It is also difficult to isolate spectral signatures directly assignable to triglycerides and fatty acids in the core of the particles from the signals that are due to the phospholipid outer shell.

To aid in the characterization of Raman spectroscopic differences between VLDLs extracted at different points in time, Raman signatures of various oil standards were acquired. Figure 3 shows the Raman spectra of three different types of fatty acids. Cis-9-octadecenoic acid is a monounsaturated fatty acid with an 18 carbon atom chain with one double bond in the cis configuration. Hexadecanoic and octadecanoic acid are both saturated fatty acids with 16 and 18 carbon atom chains, respectively. The Raman spectra of the saturated fatty acids at room temperature are noticeably different from that of the unsaturated fatty acid. Very distinct, narrow sharp peaks at $891 \mathrm{~cm}^{-1}, 1062 \mathrm{~cm}^{-1}, 1097 \mathrm{~cm}^{-1}$, $1128 \mathrm{~cm}^{-1}, 1296 \mathrm{~cm}^{-1}, 1419 \mathrm{~cm}^{-1}$, and $1440 \mathrm{~cm}^{-1}$ are observed in addition to the absence of peaks at $1266 \mathrm{~cm}^{-1}$ and $1655 \mathrm{~cm}^{-1}$. These characteristic peaks have been previously observed ${ }^{25}$ and identified in saturated triacylglycerols and saturated free fatty acids probed by Fourier transform Raman spectroscopy. The peaks, specifically at 1062,1097 , and $1128 \mathrm{~cm}^{-1}$ can be assigned to C-C stretching in a hydrocarbon chain and the $1296 \mathrm{~cm}^{-1}$ to a $\mathrm{CH}_{2}$ twist. The intensity and relative positions of the peaks in the $1000-1150 \mathrm{~cm}^{-1}$ region are sensitive to the conformational state of the hydrocarbon chain. The 1062 and $1128 \mathrm{~cm}^{-1}$ 
vibrations have been assigned to highly ordered all-trans chain segments while the $1097 \mathrm{~cm}^{-1}$ is associated with structures having gauche rotations ${ }^{18,27-29}$. A highly disordered chain results in a broadening of the gauche band and shifts to lower frequency in addition to decreases in intensity of both the 1128 and 1063 $\mathrm{cm}^{-1}$ bands. Therefore, the Raman spectra of palmitic and stearic acid at room temperature indicate that these fully saturated hydrocarbon chains are highly ordered while oleic acid, an unsaturated fatty acid with a carbon-carbon double bond results in chain disorder. If palmitic and stearic acid are heated above their melting point to induce disorder in the hydrocarbon chains, the sharp peaks that are assigned to highly ordered chains disappear and the spectra become more like that of oleic acid. Peaks due to unsaturated double bonds at $1266 \mathrm{~cm}^{-1}$ and $1655 \mathrm{~cm}^{-1}$, however, are not present. We can thus use the presence of sharp peaks in the $1000-1150 \mathrm{~cm}^{-1}$ range as markers for highly ordered saturated fatty acids, whereas peaks at $1266 \mathrm{~cm}^{-1}$ and $1655 \mathrm{~cm}^{-1}$ indicate the presence of unsaturated fatty acids.

We have obtained Raman spectra of individual VLDLs obtained from volunteers at $0 \mathrm{hr}, 3 \mathrm{hr}$, and $8 \mathrm{hr}$, respectively, after consumption of high and low glycemic load test meals. Between 20 and 30 VLDL particles were examined at each time point. All subjects were held on either high or low glycemic diets, which had been consumed for 3 days prior to the test meal. Figure 4 shows the average Raman spectra of the particles from the three time intervals for both high and low glycemic load diets. Also shown are the $3 \mathrm{hr}$ and $8 \mathrm{hr}$ difference spectra after subtraction of the $0 \mathrm{hr}$ spectra. The spectra clearly reveal differences in the 
Raman spectra at $3 \mathrm{hr}$ and $8 \mathrm{hr}$ after meal ingestion. Most notable are the formation of distinct, sharp peaks at 1060 and $1129 \mathrm{~cm}^{-1}$, a decrease in the 1266 $\mathrm{cm}^{-1}$ band, a shift and narrowing of the peak at $1298 \mathrm{~cm}^{-1}$, an increase in the $1099 \mathrm{~cm}^{-1}$ peak intensity, and a decrease in the $1650 \mathrm{~cm}^{-1}$ band for both test subjects.

Note that the $0 \mathrm{hr}$ spectra for both volunteers are very similar to those obtained from the unsaturated oleic acid. This indicates that these VLDLs are highly unsaturated and have a relative lack of any saturated fatty acids in the core of the particle. The peaks in these Raman spectra are mainly due to vibrational signatures of the phospholipid shell in addition to cholesterol ester and unsaturated fatty acids in the particle. The results from our pre- and 3 and 8 hour postprandial study of biochemical changes in VLDL particles (Figure 4) indicate that following meal consumption, the main biochemical changes in VLDLs are that the long hydrocarbon lipid chains in the particle adopt a higher ordered conformation, based on the observed changes in the $1000-1150 \mathrm{~cm}^{-1}$ region. Also, peaks due to unsaturated bonds (e.g. 1265 and $1654 \mathrm{~cm}^{-1}$ peaks associated with $\mathrm{C}=\mathrm{C}$ bonds) are decreasing in intensity for the postprandial particles.

These spectral changes reveal detailed information on the biochemical changes that are occurring in the particles as VLDL is hydrolyzed through repeated exposure to $\mathrm{LpL}$ on endothelial cell membranes. The triacylglycerols initially present in the particle are complex molecules, where the three hydrocarbon chains may consist of combinations of very different fatty acid 
chains (e.g. saturated and unsaturated chains, long and short chains, unsaturated chains with double bonds in different positions). Since it is known that saturated and unsaturated chains do not preferentially mix, instead favoring the formation of separate domains, triacylglycerol molecules encounter steric problems because the covalently linked fatty acid chains are forced to pack together. Therefore, these molecules adopt highly disordered packing. ${ }^{30-34}$ Following meal consumption, the triacylglycerol molecules packed in the particle core undergo hydrolysis as the particle interacts with endothelium and are broken down to form unsaturated and saturated free fatty acids, which are now free to form separate highly ordered domains. Accordingly, increases in the peaks in the $1000-1150 \mathrm{~cm}^{-1}$ spectral region indicate the presence of highly ordered cores of saturated fatty acids only in the 3 and 8 hour postprandial particles.

One of the main benefits of laser tweezers Raman spectroscopy is that it enables the analysis of individual particles and their statistical distributions. We can use this technique to determine particle distributions based on variations in the chemical composition from particle to particle of the same type. Here, the relative distribution of fatty acids in particles in the 3 and $8 \mathrm{hr}$ postprandial states is used to assess differences in VLDL lipolysis depending on the fat content of a meal. The normalized value of the $1060 \mathrm{~cm}^{-1}$ Raman peak from individual particle spectra was used as a marker associated with high levels of highly ordered hydrocarbon chains, which represent saturated fatty acids at room temperature. Figure $4 \mathrm{~b}$ shows a distribution plot of the saturation levels (i.e. peak intensity) of particles at 3 and 8 hours for both diets. The distribution plots clearly show that 
the saturation levels from particle to particle vary greatly for both the low and high glycemic load meals and at different times in the postprandial period. The 3 hour postprandial particles from the low glycemic study appear to have an overall higher degree of unsaturation compared to those from the high index study. This distribution shifts to the right at 8 hours, indicating a lipolysis-related increase in free saturated fatty acids with time during the postprandial state. In contrast, the particles from the high glycemic study have a similar broad distribution at both times with no significant change in the peak position between 3 and 8 hours postprandial time. One of the main differences between the two meal types is the total saturated fat content of the low and high glycemic load test meal $(16.2 \mathrm{~g}$ and $17.1 \mathrm{~g}$, respectively). It appears that the distribution analysis of the 3 hour postprandial particles directly reflects the content of saturated fatty acids of the ingested meal, while the 8 hour postprandial particle spectra no longer reflect the saturation level of the meals. For a meal with higher content of saturated fatty acids, postprandial VLDLs contain higher amounts of saturated triacylglycerols that apparently require repeated exposure to $L p L$ to convert them to free saturated fatty acids, which can then be detected spectroscopically. We believe that the reason for this observed behavior is that most of the triglycerides in the 3 hour TGRL particles still originate exogenously (i.e. from the meal) while at 8 hours, most of the triglycerides in the particles are the result of secondary liver metabolism and not remnants of exogenous triglycerides. Therefore, it is likely that the saturated triglycerides that we observe in the $8 \mathrm{hr}$ spectra represent the result of liver metabolism, containing bound fatty acids representative of de novo 
lipogenesis. Note the marked difference between the 8 hour spectra and the 0 hour spectra. The 0 hour spectra were obtained after a 12 hour fasting period. This indicates that the highly ordered saturated fatty acids found in VLDLs 8 hours after meal consumption are removed and converted some time between the 8 and 12 hour time frame. This process could be related to the transfer of fatty acids or triacylglyerols to other lipoprotein particles via transfer proteins. These plots demonstrate the ability of this technique to detect subtle changes and subdistributions in the composition of single particles that bulk or averaged spectral analysis cannot offer. However, additional experiments encompassing a larger set of data from many subjects are still required to draw definitive conclusions about these observed trends.

To further elucidate the effect of $L p L$ hydrolysis on individual VLDL particles, we exposed TGRL particles to lipoprotein lipase (LpL), an enzyme found anchored to the endothelial cell surface. Normalized Raman spectra of individual lipoprotein particles before and after exposure to lipoprotein lipase $(\mathrm{LpL})$ are shown in Figure 5. We observe a slight decrease in the $1742 \mathrm{~cm}^{-1}$ peak following hydrolysis, indicating that the triglycerides are being converted to free fatty acids. Most notable are the formation of narrow peaks at 1060, 1097, 1129, and $1298 \mathrm{~cm}^{-1}$ and a decrease in the 1265 and $1655 \mathrm{~cm}^{-1}$ peaks. Since there is no pathway for the addition of saturated fatty acids during this experiment, these spectral changes are clearly not a result of increased amounts of saturated oils in the particle but rather a direct indication of the enzymatic reaction occurring between the VLDL particle and the LpL enzyme, as discussed above. These 
changes indicate that after hydrolysis of the triacylglycerol-containing particles, the saturated fatty acids are free to form separate domains, resulting in a particle that now consists of a core with highly ordered chains. This result is very similar to the biochemical changes observed in the postprandial particles extracted after different periods in time and confirms that we are observing biochemical changes in the native VLDL particles that are due to repeated exposure to $L p L$.

\section{Conclusions}

We have, for the first time, demonstrated that individual lipoproteins can be isolated, visualized, chemically characterized, and analyzed in an entirely noninvasive fashion. Previous studies have mainly analyzed ensembles of lipoproteins. Moreover, our ex vivo analysis of individual lipoproteins can be performed rapidly and at near-physiological conditions (TGRL in pure plasma and PBS solution). This obviates the need for long, complicated and potentially destructive lipoprotein preparation protocols. This study has shown that in the population of TGRL that we analyzed by LTRS, triacylglycerols are hydrolyzed by repeated exposure to $\mathrm{LpL}$, resulting in free fatty acids. The free unsaturated and saturated fatty acids can pack into different phases, leading to the formation of a highly ordered saturated core, which can be detected spectroscopically. The particle distribution plots based on the level of highly ordered saturated fatty acids show that at the peak of the postprandial period ( 3 hours after consumption of a meal) a correlation can be made between the $\mathrm{P}: \mathrm{S}$ ratio of an ingested meal and the degree of saturation of the postprandial particles using the LTRS 
method. This result is not obvious from the averaged particle spectra shown in Fig. 3a. These results demonstrate the benefits of this single particle technique over bulk spectroscopy methods, which average over millions of particles. We expect that the combination of laser tweezers with Raman spectroscopy will ultimately allow us to follow the changes in chemical composition of a single TGRL as it is hydrolyzed by lipoprotein lipase on the endothelial cell membrane. Interactions of TGRL with endothelium can be facilitated by optically suspending the particle above a layer of endothelial cells for the Raman investigation, then deliberately initiating contact of the TGRL with the endothelial cell layer and continuing spectral interrogation during its interaction with endothelium and lipolysis by lipoprotein lipase. In addition, compositional changes in a single TGRL particle can be monitored as it is exposed to lipoprotein lipase, saturated fatty acids, and other lipids or proteins added to the buffer containing TGRL. There is considerable interest in understanding the different fatty acid conformations and complex chain ordering effects and packing, especially as it pertains to interactions within biological membranes and membrane rafts. Here, we have demonstrated such a study on chain reordering and interactions in a natural biological sample using LTRS.

\section{Acknowledgements}

This work was supported by the Richard A. and Nora Eccles Harrison Endowed

Chair in Diabetes Research, National Institutes of Health under grants HL55667 and HL71488. Dr. Motton is supported by a supplement to HL71488. The clinical 
trial was supported by funds from the U.S. Department of Agriculture, Agricultural Research Service, CRIS5330-510000-002-00D. Funding for this work at Lawrence Livermore National Laboratory was provided by the Laboratory Directed Research and Development Program. This work was also supported by funding from the National Science Foundation. The Center for Biophotonics, an NSF Science and Technology Center, is managed by the University of California, Davis, under Cooperative Agreement No. PHY 0120999. Work at LLNL was performed under the auspices of the U.S. Department of Energy by the University of California, Lawrence Livermore National Laboratory, under contract No. W-7405-Eng-48. 


\section{References}

(1) Tulenko, T. N.; Sumner, A. E. Journal of Nuclear Cardiology 2002, 9, 638649.

(2) Peticolas, W. L. Biochemical Spectroscopy 1995, 246, 389-416.

(3) Puppels, G. J.; Demul, F. F. M.; Otto, C.; Greve, J.; Robertnicoud, M.; Arndtjovin, D. J.; Jovin, T. M. Nature 1990, 347, 301-303.

(4) Molloy, J. E.; Padgett, M. J. Contemporary Physics 2002, 43, 241-258.

(5) Ashkin, A.; Dziedzic, J. M.; Bjorkholm, J. E.; Chu, S. Optics Letters 1986, 11, 288-290.

(6) Block, S. M.; Blair, D. F.; Berg, H. C. Nature 1989, 338, 514-518.

(7) Ashkin, A.; Dziedzic, J. M. Science 1987, 235, 1517-1520.

(8) Ashkin, A.; Dziedzic, J. M.; Yamane, T. Nature 1987, 330, 769-771.

(9) Ashkin, A.; Dziedzic, J. M. Proceedings of the National Academy of Sciences of the United States of America 1989, 86, 7914-7918.

(10) Bustamante, C.; Bryant, Z.; Smith, S. B. Nature 2003, 421, 423-427.

(11) Visscher, K.; Schnitzer, M. J.; Block, S. M. Nature 1999, 400, 184-189.

(12) Chan, J. W.; Esposito, A. P.; Talley, C. E.; Hollars, C. W.; Lane, S. M.; Huser, T. Analytical Chemistry 2004, 76, 599-603.

(13) Ajito, K.; Han, C. X.; Torimitsu, K. Analytical Chemistry 2004, 76, 25062510.

(14) Xie, C. G.; Li, Y. Q.; Tang, W.; Newton, R. J. Journal of Applied Physics 2003, 94, 6138-6142.

(15) Xie, C. G.; Li, Y. Q. Journal of Applied Physics 2003, 93, 2982-2986.

(16) Xie, C. G.; Dinno, M. A.; Li, Y. Q. Optics Letters 2002, 27, 249-251.

(17) Cherney, D. P.; Bridges, T. E.; Harris, J. M. Analytical Chemistry 2004, 76, 4920-4928.

(18) Cherney, D. P.; Conboy, J. C.; Harris, J. M. Analytical Chemistry 2003, 75, 6621-6628.

(19) Sanderson, J. M.; Ward, A. D. Chemical Communications 2004, 11201121.

(20) Verma, S. P.; Philippot, J. R.; Bonnet, B.; Saintemarie, J.; Moschetto, Y.; Wallach, D. F. H. Biochemical and Biophysical Research Communications 1984, 122, 867-875.

(21) Fraile, M. V.; Carmona, P. Spectrochimica Acta Part a-Molecular and Biomolecular Spectroscopy 1996, 52, 557-563.

(22) Lopez, G.; Martinez, R.; Gallego, J.; Tarancon, M. J.; Carmona, P.; Fraile, M. V. Applied Spectroscopy 2000, 54, 1771-1776.

(23) Vlasova, I. M.; Dolmatova, E. V.; Koshelev, V. B.; Saletsky, A. M. Laser Physics Letters 2004, 1, 417-420.

(24) Frank, C. J.; Redd, D. C. B.; Gansler, T. S.; Mccreery, R. L. Analytical Chemistry 1994, 66, 319-326.

(25) Weng, Y. M.; Weng, R. H.; Tzeng, C. Y.; Chen, W. L. Applied Spectroscopy 2003, 57, 413-418. 
(26) Baeten, V.; Hourant, P.; Morales, M. T.; Aparicio, R. Journal of Agricultural and Food Chemistry 1998, 46, 2638-2646.

(27) Brown, K. G.; Peticolas, W. L.; Brown, E. Biochemical and Biophysical Research Communications 1973, 54, 358-364.

(28) Spiker, R. C.; Levin, I. W. Biochimica et Biophysica Acta 1975, 388, 361373.

(29) Gaber, B. P.; Peticolas, W. L. Biochimica et Biophysica Acta 1977, 465, 260-274.

(30) Kaneko, F.; Yano, J.; Sato, K. Current Opinion in Structural Biology 1998, 8, 417-425.

(31) Small, D. M. Journal of Lipid Research 1984, 25, 1490-1500.

(32) Di, L.; Small, D. M. Journal of Lipid Research 1993, 34, 1611-1623.

(33) Di, L.; Small, D. M. Biochemistry 1995, 34, 16672-16677.

(34) Small, D. M. Current Opinion in Structural Biology 1998, 8, 413-416. 
Table I: Raman frequencies of individual TGRL and their assignments

\begin{tabular}{cl}
\hline $\begin{array}{c}\text { Raman frequency in } \\
\text { wavenumber units }\left(\mathrm{cm}^{-1}\right)\end{array}$ & \multicolumn{1}{c}{ Assignment $^{\mathrm{a}}$} \\
\hline 727 & $\delta(=\mathrm{C}-\mathrm{H})$ in-plane \\
972 & $\delta(=\mathrm{C}-\mathrm{H})$ out-of-plane \\
1066 & $v(\mathrm{C}-\mathrm{C})$ \\
1079 & $v(\mathrm{C}-\mathrm{C})$ \\
1119 & $v(\mathrm{C}-\mathrm{C})$ \\
1265 & $\delta(=\mathrm{C}-\mathrm{H})$ in-plane cis \\
1302 & $\delta\left(\mathrm{CH}_{2}\right)$ twisting \\
1439 & $\delta\left(\mathrm{CH}_{2}\right)$ scissor \\
1455 & $\delta\left(\mathrm{CH}_{2}\right)$ \\
1654 & $v(\mathrm{C}=\mathrm{C})$ cis $($ lipid $)$, and $v(\mathrm{O}-\mathrm{H})$ (water $)$ \\
1742 & $v(\mathrm{C}=\mathrm{O})$ in $-\mathrm{CH}_{2}-\mathrm{COOR}$ \\
\hline
\end{tabular}

${ }^{a}$ Abbreviations: $v$ and $\delta$ indicate stretching and deformation vibrations, respectively. See text for more details. Assignments are based on $\left[{ }^{24},{ }^{25}\right]$. 


\section{Figure Legends}

Figure 1. (a) Confocal spectroscopy system for the detection of the Raman spectra of single TGRL particles (b) Optical trapping of a single TGRL particle in plasma solution and simultaneous spectral acquisition using a single laser beam focused through a high numerical aperture objective.

Figure 2. Raman spectrum of a single optically trapped TGRL particle taken with $10 \mathrm{~mW}$ laser power for 60 seconds (see table for peak assignments). A particle is confirmed to be trapped by detection of the backscattered laser light off the particle at the focus (see inset).

Figure 3. Raman spectra of oleic, palmitic, and stearic acid at (a) room temperature and (b) $70^{\circ} \mathrm{C}$. Saturated fatty acids (palmitic and stearic acids) at room temperature exhibit a number of unique sharp spectral peaks, e.g. at 1062, 1128 , and $1296 \mathrm{~cm}^{-1}$ that are not observed for the unsaturated oleic acids. These changes indicate highly ordered carbon chains in the saturated fatty acids, which disappear at temperatures above the melting point (b). In this case, the $C=C$ vibrations at $1266 \mathrm{~cm}^{-1}$ and $1655 \mathrm{~cm}^{-1}$ become the most distinct differences between the fatty acid standards.

Figure 4. (a) Averaged Raman spectra of TGRL particles extracted from human volunteers at three time intervals before and after the consumption of a meal, and the 3 and 8 hour difference spectra. (b) Plots showing the particle distribution based on the intensity of the $1060 \mathrm{~cm}^{-1}$ peak, indicative of the concentration of highly ordered saturated fatty acids in the particles. Solid lines are fits to the particle distributions and serve only as guides to the eye.

Figure 5. Individual Raman spectra of TGRL particles before and after 1 hour exposure to lipoprotein lipase. The Raman spectrum of lipoprotein lipase is also shown. 


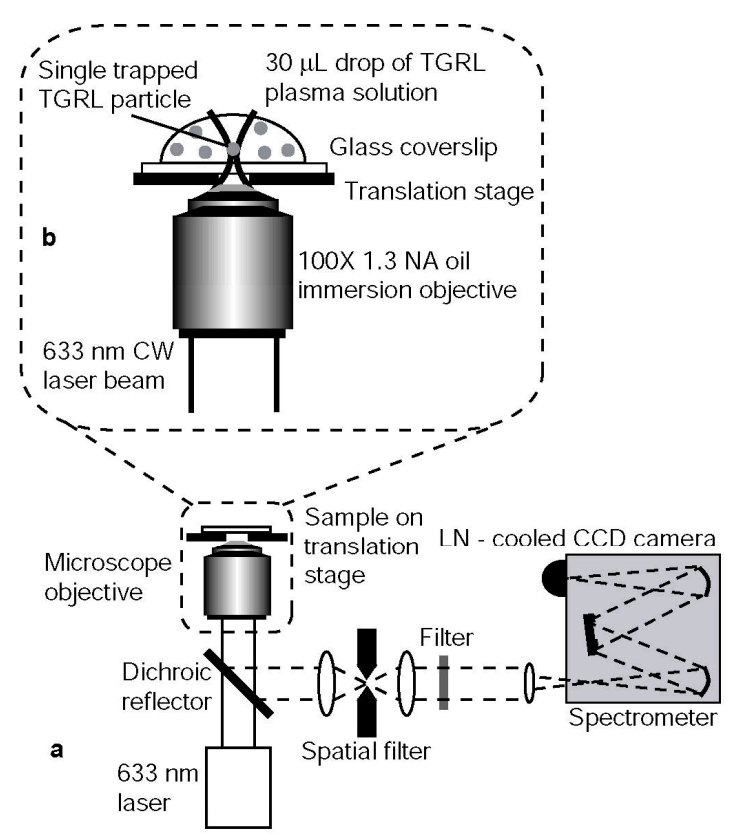

Figure 1. (Chan). (a) Confocal spectroscopy system for the detection of the Raman spectra of single TGRL particles (b) Optical trapping of a single TGRL particle in plasma solution and simultaneouos spectral acquisition using a single laser beam focused through a high numerical aperture objective 


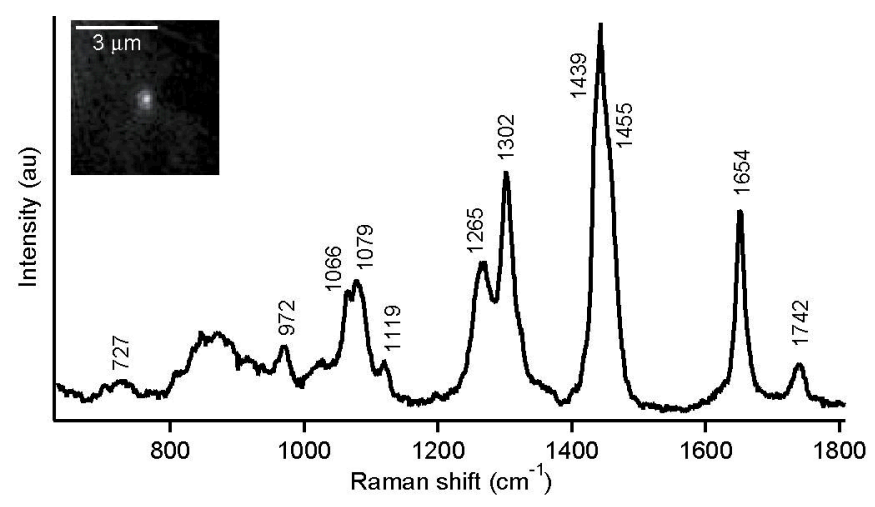

Figure 2. (Chan). Raman spectrum of a single optically trapped TGRL particle taken with $10 \mathrm{~mW}$ laser power for 60 seconds (see table for peak assignments). A particle is confirmed to be trapped by detection of the backscattered laser light off the particle at the focus (see inset). 


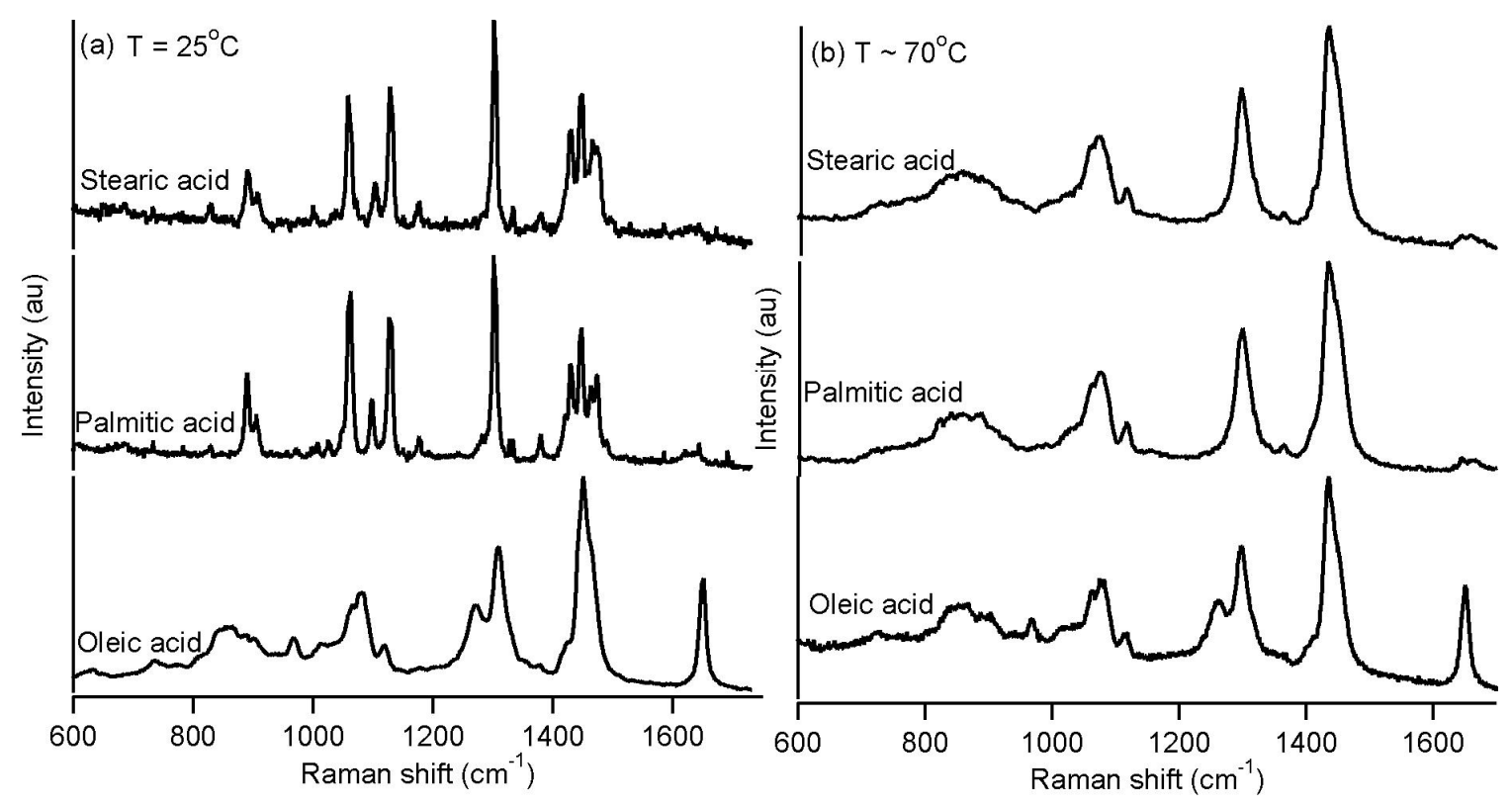

Figure 3. (Chan) Raman spectra of oleic, palmitic, and stearic acid at (a) room temperature and (b) 70 degrees Celsius. Saturated fatty acids (palmitic and stearic acids) at room temperature exhibit a number of unique sharp spectral peaks, e.g. at 1062, 1128, and $1296 \mathrm{~cm}-1$ that are not observed for the unsaturated oleic acids. These changes indicate highly ordered chains in the saturated fatty acids, which disappear at temperatures above the melting point (b). In this case, the $\mathrm{C}=\mathrm{C}$ vibrations at 1266 and $1655 \mathrm{~cm}-1$ become the most distinct differences between the fatty acid standards. 

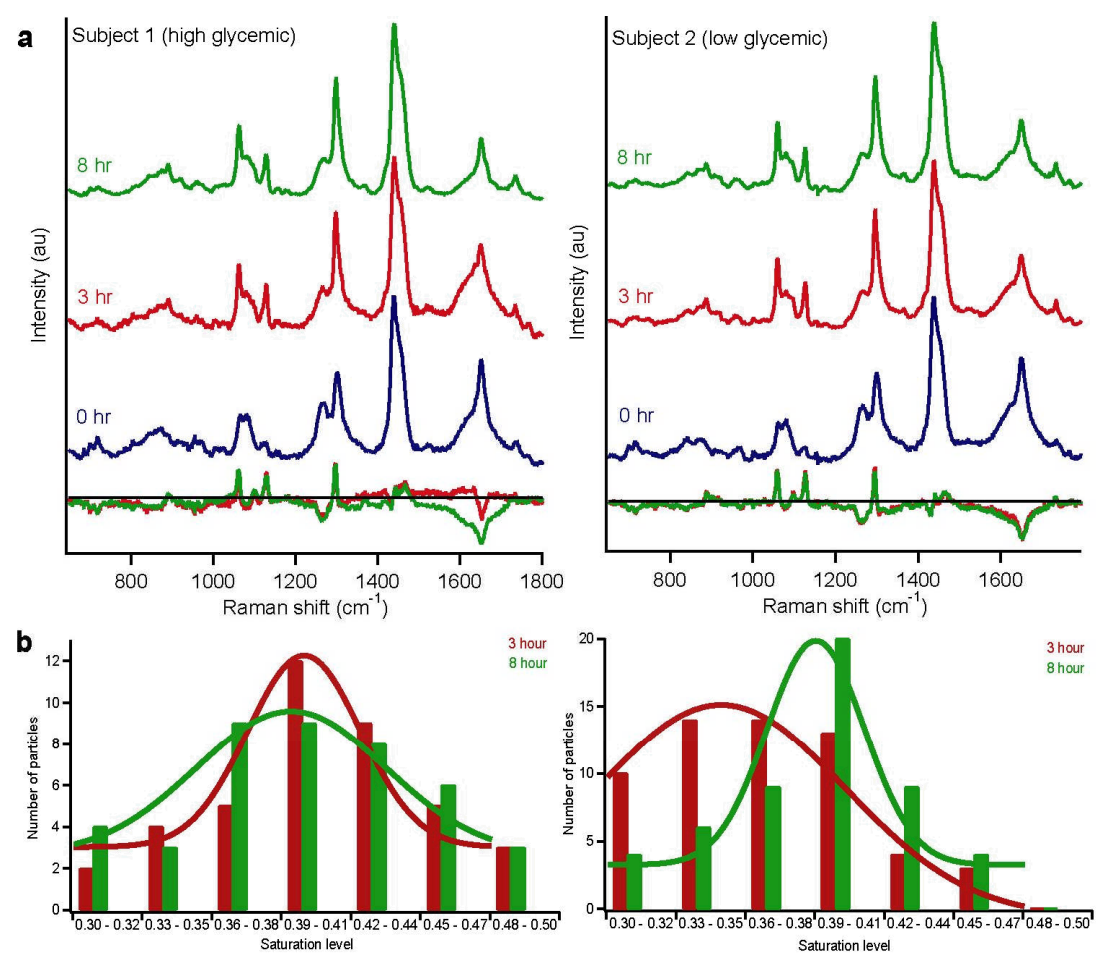

Figure 4. (Chan). (a) Averaged Raman spectra of TGRL particles extracted from human volunteers at three time intervals before and after consumption of a meal, and the 3 and 8 hour difference spectra (b) Plots showing the particle distribution based on the based on the intensity of the $1060 \mathrm{~cm}-1$ peak, indicative of the concentration of highly ordered saturated fatty acids in the particles. Solid lines are fits to the particle distributions and serve only as guides to the eye. 


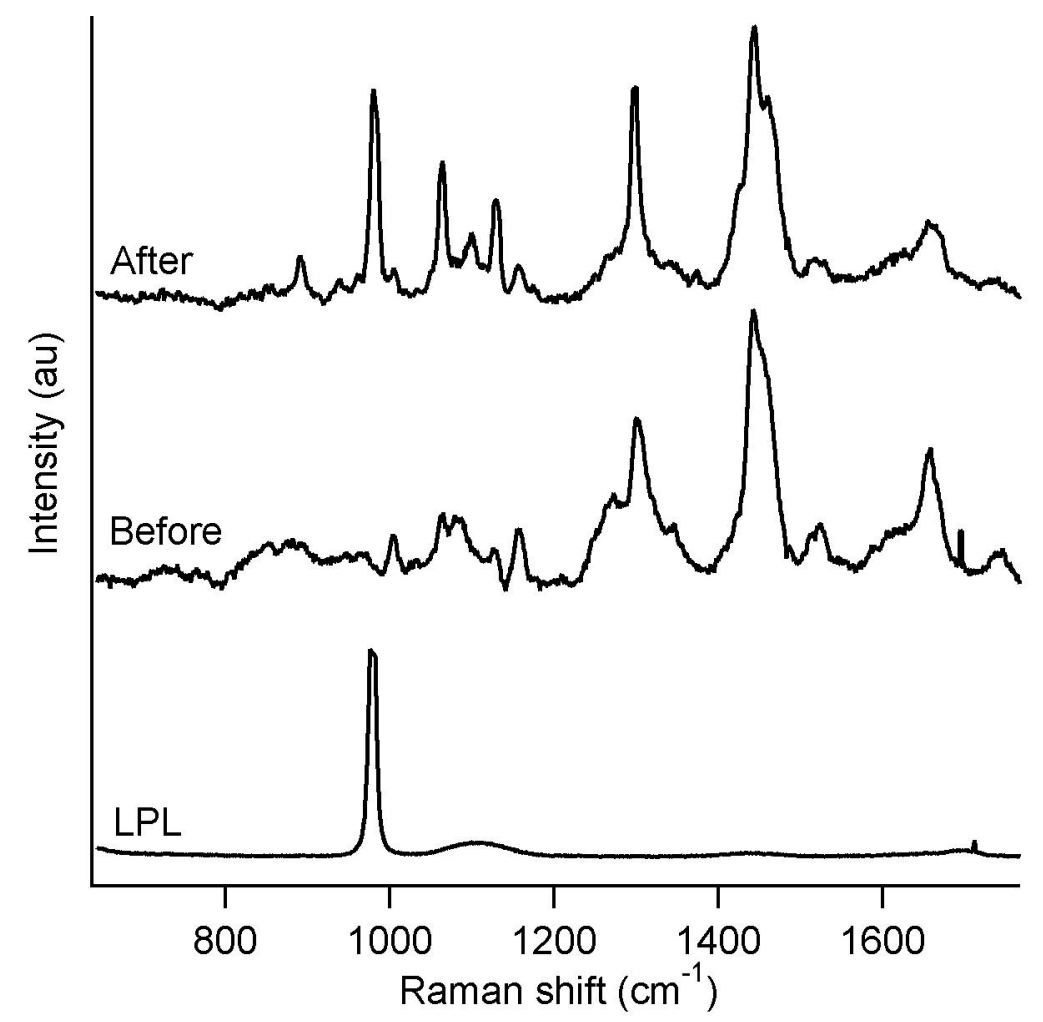

Figure 5. (Chan). Individual Raman spectra of TGRL particles before and after 1 hour exposure to lipoprotein lipase. The Raman spectrum of lipoprotein lipase is also shown. 